\title{
ALIMENTOS DEL FUTURO: LA CIENCIA FICCIÓN CULINARIA A TRAVÉS DEL ARTE
}

\author{
Francisca Ramón Fernández a, Cristina Lull Noguera b ${ }^{b}$ María Desamparados \\ Soriano Soto ${ }^{c}$ \\ a Departamento de Urbanismo. Universitat Politécnica de Valencia. frarafer@urb.upv.es \\ ${ }^{b}$ Departamento de Química. Universitat Politécnica de Valencia. clull@upvnet.upv.es \\ c Departamento de Producción Vegetal. Universitat Politécnica de Valencia. asoriano@prv.upv.es
}

\begin{abstract}
The proposal related to the study of different works of art is presented in which the socalled "futuristic food" is reflected. After the publication in 1930 of the Manifesto of Futurist Cooking, in which a gastronomic revolution began with the appearance of dishes such as "Pollo Fiat", "Carneplástico", or sensory experiences such as "Aeroabanquete", there are numerous occasions in which the avant-garde in gastronomy has been reflected in an artistic work, mainly in painting. Based on this consideration we will analyze from different points of view such well-known works as Campbell's soup cans, by Andy Warhol; Nedick's, 1970, by Richard Estes, Floor Burger, by Claes Oldenburg; Cakes, by Wayne Thibaud; Ham and egg sandwich, Tjalf Sparnaay, or Figure with meat, by Francis Bacon. From the aforementioned analysis, valid conclusions have been obtained regarding the use of food in the pictorial work, and its social connotation and sensitive perspective, in addition to its influence on the time in which it is located.
\end{abstract}

Keywords: futuristic food, museum.

Resumen: Se presenta la propuesta relativa al estudio de distintas obras de arte en las que se plasma la denominada "comida futurista". Tras la publicación en 1930 del Manifiesto de la Cocina Futurista, en la que se inicia una revolución gastronómica con la aparición de platos como el "Pollo Fiat", el "Carneplástico", o experiencias sensoriales como el "Aeroabanquete", son numerosas las ocasiones en las que la vanguardia en la gastronomía se ha plasmado en una obra artística, principalmente en la pintura. Partiendo de esta consideración analizaremos desde distintos puntos de vista obras tan conocidas como las latas de sopa Campbell, de Andy Warhol; Nedick's, 1970, de Richard Estes, Hamburguesa de piso, de Claes Oldenburg; Pasteles, de Wayne Thibaud; Sándwich de jamón y huevo, Tjalf Sparnaay, o Figura con carne, de Francis Bacon. Del análisis indicado se han obtenido unas conclusiones válidas respecto a la utilización de los alimentos en la obra pictórica, y su connotación social y perspectiva sensitiva, además de su influencia en la época en la que se sitúa la misma.

Palabras clave: comida futurista, museos.

Citar como: Ramón Fernández, F., Lull Noguera, C., Soriano Soto, M. D. (2022). "Alimentos del futuro: la ciencia ficción culinaria a través del arte". En: Actas del III Congreso Internacional sobre Patrimonio Alimentario y Museos. 25-26 noviembre, 2021, Valencia, España. pp. 85-97. https://doi.org/10.4995/EGEM2021.2021.13927 


\section{Introducción}

La comida ha constituido una fuente de inspiración para los artistas, quienes según la época la han interpretado desde perspectivas muy distintas, dando como resultado obras categóricamente opuestas. Todos los atractivos que los alimentos tuvieron para los antiguos creadores siguen vigentes en las vanguardias y llega hasta el arte más actual de la mano de artistas que otorgan a estos materiales dimensiones nuevas y dispares que alientan nuevos relatos

Desde la pintura meramente ilustrativa o documental que nos muestra maneras de cocinar, platos típicos y banquetes referentes de épocas como la egipcia o la romana se ha realizado un viaje por la representación del alimento en las distintas religiones. Ya en los siglos XVI y XVII la llegada del bodegón haría que ésta saltara a un primer plano y se convirtiera en la protagonista de la escena. Pero dependiendo de la época de la obra, la comida se ha introducido con diversas intenciones, ya fuera como indicativo de una posición social en Vermeer o Van Gogh, como elemento caricaturesco de sátira o ironía en los geniales retratos de Arcimboldo o a modo de única protagonista de la composición ya en bodegones llenos de detalle como los de Luis Meléndez o Paul de Vos. Posteriormente, Cézanne, fue precursor de las formas abstractas, más tarde Picasso presentará otra evolución en los objetos y alimentos de estas naturalezas muertas. Autores como Charlesp, Gauguin, Matisse, Salvador Dalí, Juan Gris o Luis Fernández se servirán también de la potencia expresiva de la comida y sus elementos para plasmar sus imaginarios en el soporte artístico.

Finalmente la tendencia tiende a incluir platos concebidos en impresoras 3D, granjas verticales, microencapsulados con nutrientes decisivos, hongos que curan el medio ambiente... etc.

Es precisamente tras la publicación del Manifiesto de la Cocina Futurista, en 1930, en las que aparecen platos como el Pollo Fiat, el Carneplástico o experiencias sensoriales como el Aerobanquete, que hacen replantearse el concepto del alimento y se genera una discusión sobre el futuro de la alimentación influida por la ciencia ficción.

Nuestro objeto en este trabajo es estudiar la relación de la comida futurista con el arte mediante el estudio de distintas obras de arte en las que en ocasiones la vanguardia en la gastronomía se ha plasmado en una obra artística, como las latas de sopa Campbell, de Andy Warhol; Nedick's, 1970, de Richard Estes, Hamburguesa de piso, de Claes Oldenburg; Pasteles, de Wayne Thibaud; Sándwich de jamón y huevo, de Tjalf Sparnaay, o Figura con carne, de Francis Bacon. Junto a ello, estos ejemplos pueden servirnos para una innovación docente en la asignatura de Derecho alimentario y protección del consumidor, en la titulación del Grado en Ciencia y Tecnología de Alimentos, ya que se muestra la evolución del alimento y su representación gráfica.

La metodología que vamos a emplear es el análisis de las distintas obras pictóricas seleccionadas, así como el estudio de los alimentos del futuro, en un escenario de ciencia ficción mediante las aportaciones doctrinales más recientes.

Del análisis indicado se han obtenido unas conclusiones válidas respecto a la utilización de los alimentos en la obra pictórica, y su connotación social y perspectiva sensitiva, además de su influencia en la época en la que se sitúa la misma. 


\section{Los alimentos del futuro: un escenario de ciencia ficción}

El futurismo es una corriente artística contemporánea de principios del siglo XX, se creó como movimiento artístico y cultural y se abre a todas las formas de expresión: pintura, escultura, literatura, música, arquitectura, danza, fotografía, cine y, gastronomía. Se cree que el futurismo italiano fue el único movimiento artístico que se atrevió a repensar la tradición culinaria de su país, el único que elaboró "un programa de renovación total de cocinas"

Para los futuristas, cocinar era una forma de expresión artística, que ofrecían platos que definían apetitosos complejos plásticos, o esculturas para comer. Podríamos resumir la relación entre futurismo y cocina diciendo que en todos los aspectos la cocina era una expresión artística. El plato primero debe ser hermoso a la vista, con combinaciones de colores particulares.

Los problemas actuales incluido el covid19 junto al cambio climático, y el agotamiento de recursos están originando una transformación que hace más eficiente el cultivo de productos vegetales, la producción de alimentos más respetuosos con el medio ambiente y el bienestar animal, el favorecimiento de la acuicultura como alternativa a la sobreexplotación de los mares. Y la más extrema que es la obtención de insectos para el consumo humano.

Las microcápsulas es una de las revoluciones que procede del microencapsulado, un conjunto de técnicas mediante las cuales en pequeñas partículas, gotas de líquido o gases da lugar a microcápsulas o partículas agregadas para desarrollar alimentos más seguros, con mayor extensión de su vida útil, más saludables y con nuevas propiedades. Aunque parece, no es ciencia ficción: la microencapsulación permite mantener la estabilidad de nutrientes como la vitamina C (una proteína decisiva en la producción y regeneración de piel, tendones, ligamentos y vasos sanguíneos) o los omega 3 (ácidos grasos trascendentes para fortalecer las neuronas y mantener el corazón sano) o incorporar calcio en bebidas vegetales.

Daniel Rivera, responsable de Tecnologías de Microencapsulación de Ainia, un centro tecnológico de referencia en $1+d+i$ alimentaria asegura que el futuro no se puede concebir sin esta revolución. "Esta tecnología transcurre paralela al desarrollo de nuevos materiales que permitan cumplir con más requerimientos, soportar mejor determinadas condiciones y liberarse en aspectos y límites concretos", como aumentar su vida útil, prolongando la fecha de caducidad, y contribuir a un menor desperdicio de alimentos, o permitir flexibilizar condiciones de conservación (por ejemplo, reduciendo su permanencia en sistemas de refrigeración).

La metagenómica y la microbiología tradicional posibilita el control sanitario de los alimentos evitando microorganismos patógenos como la Salmonella y abre camino como una técnica que permite un examen exhaustivo de los patógenos existentes para identificar qué especies microbianas están presentes y en qué porcentaje, gracias a un análisis de ADN en la muestra para analizar. Los resultados son mucho más completos que en un análisis microbiológico, porque ofrecen información sobre todos los patógenos existentes en la muestra, y no solo sobre las bacterias que se buscan en el otro sistema.

Carne de laboratorio como hamburguesas que evitan el consumo de carne de ganado y el abuso de la ganadería. Hamburguesa vegetal que parece de carne y al cocinar suelta liquido parecido a la sangre que es el jugo de la remolacha que lleva. Carnes in vitro, creadas con células madres y sin productos químicos, aún está en proceso, buscando cómo reducir costes, Cuhi con algas sustitutivo a la ensalada con pescado fresco marinado, comidas líquidas y en barrita con todos los nutrientes necesarios. 
Videos como La Comida del Futuro - Bing vídeo de planeta vivo, inventos como burbuja de agua, helado negro hecho de carbón y almendras en una heladería de Los Ángeles, café transparente que protege la placa dental sin dañarla, evitando manchas.

Dentro de la biotecnología fúngica las setas y los hongos tienen un poder más allá de la temporada de cesta y mercado. Estos frutos del campo protagonizan una importante línea de investigación para dar respuesta al desarrollo de una industria alimentaria con capacidad para proporcionar sustento a todo el planeta, a partir de la eliminación de daños medioambientales. La clave de la biotecnología fúngica está en el ciclo de vida de los filamentos de los hongos y de sus transformaciones celulares partiendo de las esporas, una tecnología reconocida como motor de innovación dentro de los Objetivos de Desarrollo Sostenible de la ONU, con múltiples aplicaciones en la industria química, textil y alimentaria.

Hay proyectos como el denominado PECOSAIN, llevado a cabo por AZTI - un centro científico y tecnológico que desarrolla proyectos de transformación de alto impacto-, que persigue mejorar la calidad de los productos de pescado mediante la generación - vía biotecnología fúngica- de coberturas (empanados y/o rebozados) innovadoras, crujientes y saludables. Y a más corto plazo, un proyecto que ya desarrolla el Instituto de Agroquímica y Tecnología de Alimentos del Consejo Superior de Investigaciones Científicas (CSIC), con aplicación en el campo de la enología: se desarrollan vinos con mejores condiciones organolépticas o funcionales.

La revolución de las máquinas descubre a tiempo real defectos en pleno proceso de producción y favorecerá la reducción de excedentes de producción y otorgar a las empresas un conocimiento profundo sobre los alimentos.

¿Nutrición individualizada con alimentos impresos en 3D? Algunos investigadores están creando estos productos. La población mundial está envejeciendo rápidamente y existe una necesidad urgente de analizar las necesidades de alimentos y nutrición para este sector de la sociedad, se espera que la proporción de personas de más de 65 años haya aumentado del 25 al $40 \%$ en 2030.

Estrategias que se verán aceleradas con el nuevo programa de I+D+i de la Unión Europea denominado Horizonte Europa 2021-2027, que busca impulsar la ciencia abierta, la competitividad industrial y la innovación, con especial atención a aspectos de nuestra vida cotidiana como la salud, el medio ambiente y la alimentación. En definitiva, una evolución de la comida cuyo respaldo a través de tecnologías como la impresión 3D, el loT (Internet de las cosas, integración de todo tipo de objetos informáticos o electrónicos a través de la red gracias a sensores) o el big data (el poder que otorgan los datos de los usuarios) permiten transformar nuestros menús a un ritmo de vértigo.

La disfagia afecta a más de un tercio de ancianos y deteriora su capacidad de consumir alimentos normales, motivo por el que las personas mayores a menudo rechazan las comidas, comen porciones muy pequeñas y prefieren alimentos fáciles de comer, en detrimento de aquellos que les proporcionan los nutrientes que necesitan en las cantidades adecuadas. La textura se adapta para ser más fácil de tragar y también permite calibrar la cantidad de azúcar, sal y grasas, ya que la preparación de la comida sigue siendo de la forma tradicional. La idea es que platos como la carne de cerdo con patatas, se puedan llegar a imprimir a gran escala directamente en un plato con las impresoras 3D ubicadas en las cocinas de las residencias de ancianos. 
Granjas verticales: agricultura a ras del cielo. La afectación del cambio climático sobre los cultivos, junto a la creciente demanda de productos consecuencia del crecimiento de la población, exigen nuevas formas de abordar la agricultura. Una de las propuestas más prometedoras son las granjas verticales, grandes extensiones de cultivo de plantas dentro de edificios denominados farmscapers, que funcionan con la mayor eficiencia energética. Son verdaderos museos de cultivos controlados y protegidos.

Eso es posible utilizando la energía LED (Light Emiting Diode, diodo emisor de luz) como herramienta de fotosíntesis y su cultivo en hidropónico (en una solución mineral, en lugar de en suelo agrícola). A su vez, se consigue reducir un $95 \%$ el consumo de agua con respecto a un cultivo tradicional. Además, la automatización del big data evita depender de las inclemencias climáticas y tener varias cosechas al año de productos de calidad y cercanía. Sin embargo, las críticas a este sistema surgen de la desvinculación de la agricultura del entorno rural.

Uno de los proyectos más emblemáticos es Aerofarms, en Nueva York: desde un edificio de 6.500 metros cuadrados se producen 900 toneladas de verduras de hoja al año. Europa tiene ya varios planes señeros en agricultura vertical y, en el caso de España, este novedoso sistema empieza a dar los primeros pasos. Entre sus grandes impulsores se encuentran gigantes tecnológicos como Google o Tesla, así como países de Oriente Medio.

El consumo de algas no es tradicional en nuestro país, pero en la globalización, se produce al auge de la gastronomía japonesa y cada vez mayor disponibilidad de productos en las tiendas, las algas se van haciendo un hueco en la dieta de los españoles. Delicias como los maki rolls de sushi, la ensalada sunomomo o la sopa de miso contienen algas como ingrediente importante, y son platos de mucho éxito. Esta fama se ha reforzado por cierta corriente que sitúa a las algas como el "superalimento" de moda, atribuyéndoles todo tipo de propiedades y recomendando su consumo habitual. Pero ¿es acertado? Estas "verduras" marinas no son un alimento insano, pero pueden no ser seguras en dosis altas por algunas de sus características, como su elevado contenido en yodo.

En el contexto del creciente interés de la población referente a la salud y el bienestar, los alimentos funcionales (surgidos en los años 80, en Japón) juegan un papel clave en la dieta. Esta tendencia hace que la producción de ingredientes funcionales, con actividad demostrada y estabilizada para su incorporación a un mayor número de matrices de alimentos, sea un área de estudio de gran interés y muy demandado por la industria de alimentaria.

Reducir la contaminación de los suelos es otro de los objetivos del programa Horizonte Europa, que fija como meta la reducción de pesticidas y fertilizantes en los cultivos del continente. Gemma del Caño, experta en I+D e Industria, recuerda que, en general, los fitosanitarios son necesarios para evitar plagas o microorganismos que estropeen la producción, por lo que hay que intentar que contaminen lo menos posible. "Sería fantástico no tener que utilizar ninguno, pero eso va a llevar tiempo", explica. "También es muy importante diferenciar entre los problemas que pueden provocar en su entorno laboral -dadas las investigaciones recientes sobre el riesgo de cáncer de los trabajadores agrícolas al entrar en contacto con ciertos herbicidas y los que llegan al consumidor, que nada tienen que ver. Hoy podemos decir que los fitosanitarios no están en los alimentos en cantidades perjudiciales para las personas".

En los próximos siete años, el consorcio europeo EIT Food, compuesto por 50 empresas, universidades y centros de investigación, invertirá cerca de 1.600 millones de euros para desarrollar 
los alimentos del futuro: impulsará la transformación del sector alimentario centrándose en el consumidor y en la eficiencia de los recursos. Ajustar la producción alimentaria a las nuevas necesidades y adaptarla para utilizar menos materias primas y reducir los desperdicios.

Si hablamos de moléculas bioactivas debemos recurrir al trabajo de Aora Health y su amplio catálogo de nutracéuticos de última generación, que combina para generar efectos sinérgicos positivos junto con una aproximación científica que de la selección de los ingredientes adecuados, el control de la cantidad de molécula absorbida por el organismo (biodisponibilidad). Entre sus componentes destacan el AOX® (Extracto de Amla-Extracto de Polygonum-Extracto de Zanahoria), el Extracto de Pasiflora, el Extracto de Amapola, el Extracto de Azafrán, la Vitamina B6 y la Melatonina.

¿Cómo serán los alimentos dentro de 25 o 50 años? ¿Y dentro de un siglo? ¿Triunfarán los insectos? ¿La comida se administrará en píldoras? ¿Podremos comer todo lo que queramos y no engordar un gramo?

Los cuadros, las películas y las novelas de ciencia ficción contienen algunas propuestas interesantes. De momento, pensamos que los cambios no serán tan drásticos, lo único que se puede avanzar en el futuro más próximo, que será una continuación de las tendencias actuales.

Pero sí que aparecerá la búsqueda de nuevas zonas de cultivo, existen propuestas de cultivar en Marte, ¿primero habrá que llegar no?, pero como ya se conocen las condiciones se están preparando cultivos aptos para su desarrollo en Marte cuando se pueda llegar (National Geographic, 2016). Dentro del proyecto SomosFuturo que impulsa el talento de los jóvenes implicando la nanotecnología para el cultivo de granjas inteligentes utilizando robots. Experiencias en países bajos han tenido éxito con 9 verduras y cereales.

También interesa destacar la figura de Ángel León, que al mando de su restaurante Aponiente, desarrolló plancton para el consumo humano, y que recientemente ha mostrado su descubrimiento con un cereal marino sostenible cultivado (Fuchs, 2021).

\section{Los alimentos del futuro en el arte: principales obras pictóricas y escultóricas en la que se plasma una visión de ciencia ficción}

Son muchas las obras pictóricas que representan un alimento o comida. Los bodegones son muy habituales y han sido representados a lo largo del tiempo. La comida ha sido considerada como un espacio esencial dentro del arte contemporáneo (Díaz Ruiz, 2017).

El movimiento futurista que se inició con Filippo Tommaso Emilio Marinetti, con la publicación de su Manifiesto futurista, de 1909, en el que propugnaba una exaltación al amor, al liderazgo, al peligro y la agresividad y que influyó en el ámbito del arte. Uno de sus representantes más conocidos fue Giacomo Balla que se adhirió al movimiento con la firma de La pittura futurista Manifesto técnico, en 1910.

Marinetti, en 1931 abrió un restaurante llamado El Paladar Sagrado, que se caracterizaba por una decoración fría y metálica, y en el que los platos que se servían eran manifestaciones de ese futurismo (Miño, 2020). 


\subsection{Las latas de Sopas Campbell, de Andy Warhol}

Una de las primeras obras seleccionadas son las Latas de sopa Campbell's, 1962, de Andy Warhol (Figura 1), realizada en pintura de polímero sintético sobre tela, 50,8 cm x 40,6 cm. y que se encuentra en el Museum of Modern Art, más conocido por su acrónimo MoMA, en Nueva York. Se trata de una obra representativa del denominado pop art.

Es un icono dentro del arte, y contempla la comida enlatada, como símbolo del futuro, con una clara intención de facilitar un consumo a futuro de un alimento, ya que se incrementa la conservación a través del enlatado.

\subsection{Nedick's, 1970, de Richard Estes}

La obra de Richard Estes que se encuentra en el Museo Nacional Thyssen-Bornemisza, es un óleo sobre lienzo, 121,9 x 167,6 cm, y pertenece a la Colección Carmen Thyssen-Bornemisza (Figura 2).

Esta obra es representativa de la denominada "comida rápida", ya que representa un paisaje urbano muy característico de la ciudad de Nueva York. Se observa la presencia de alimentos como las hamburguesas y los hot dog (perritos calientes), además de zumos de frutas. Denota una comida que actualmente es muy consumida principalmente por el público adolescente, por la rapidez y las connotaciones sociales. Importante hacer mención del logotipo del refresco, Coca Cola, que está asociada a la modernidad. Esta obra se enmarca dentro del movimiento del Fotorrealismo.
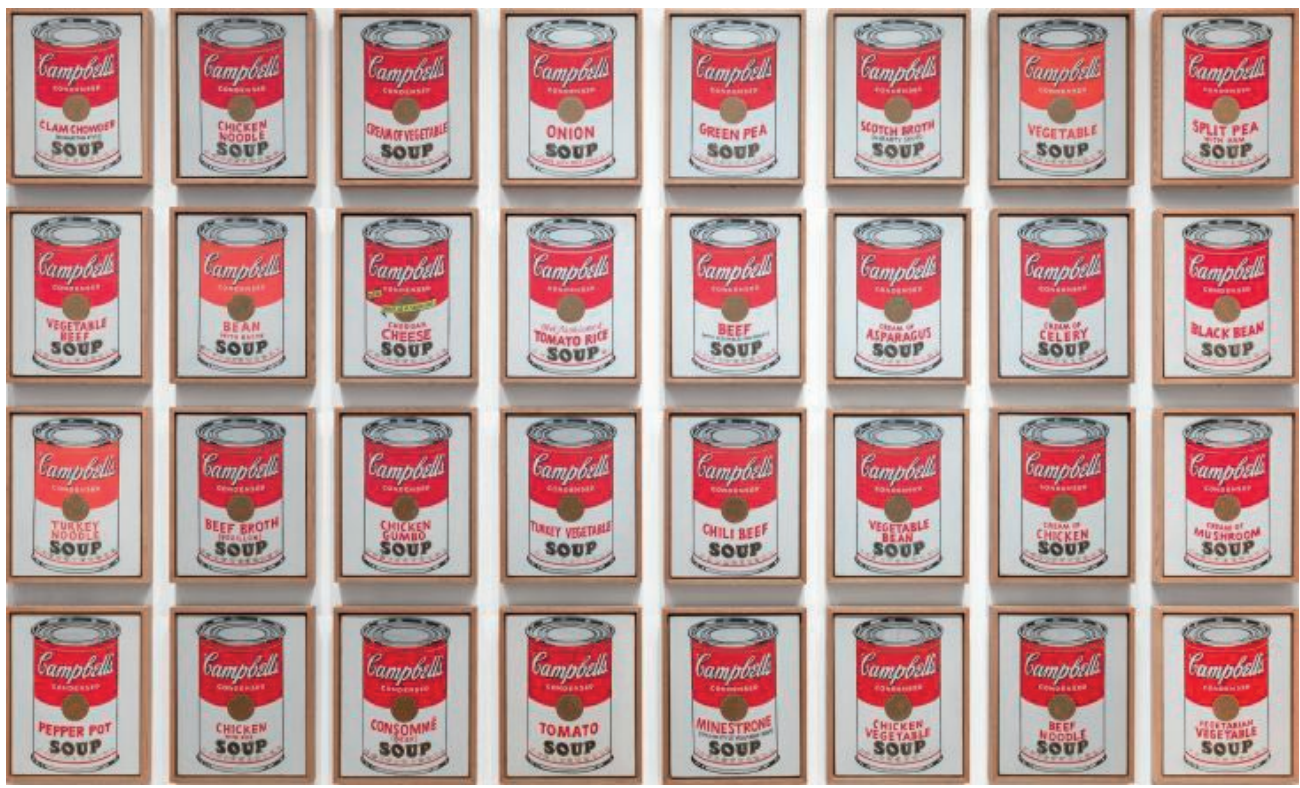

Figura 1. Imagen de Latas de sopa Campbell's, de Andy Warhol (1962).

Fuente: 3minutos de arte: https://3minutosdearte.com/cuadros-fundamentales/latas-de-sopa-campbells-1962-andy-warhol/ 


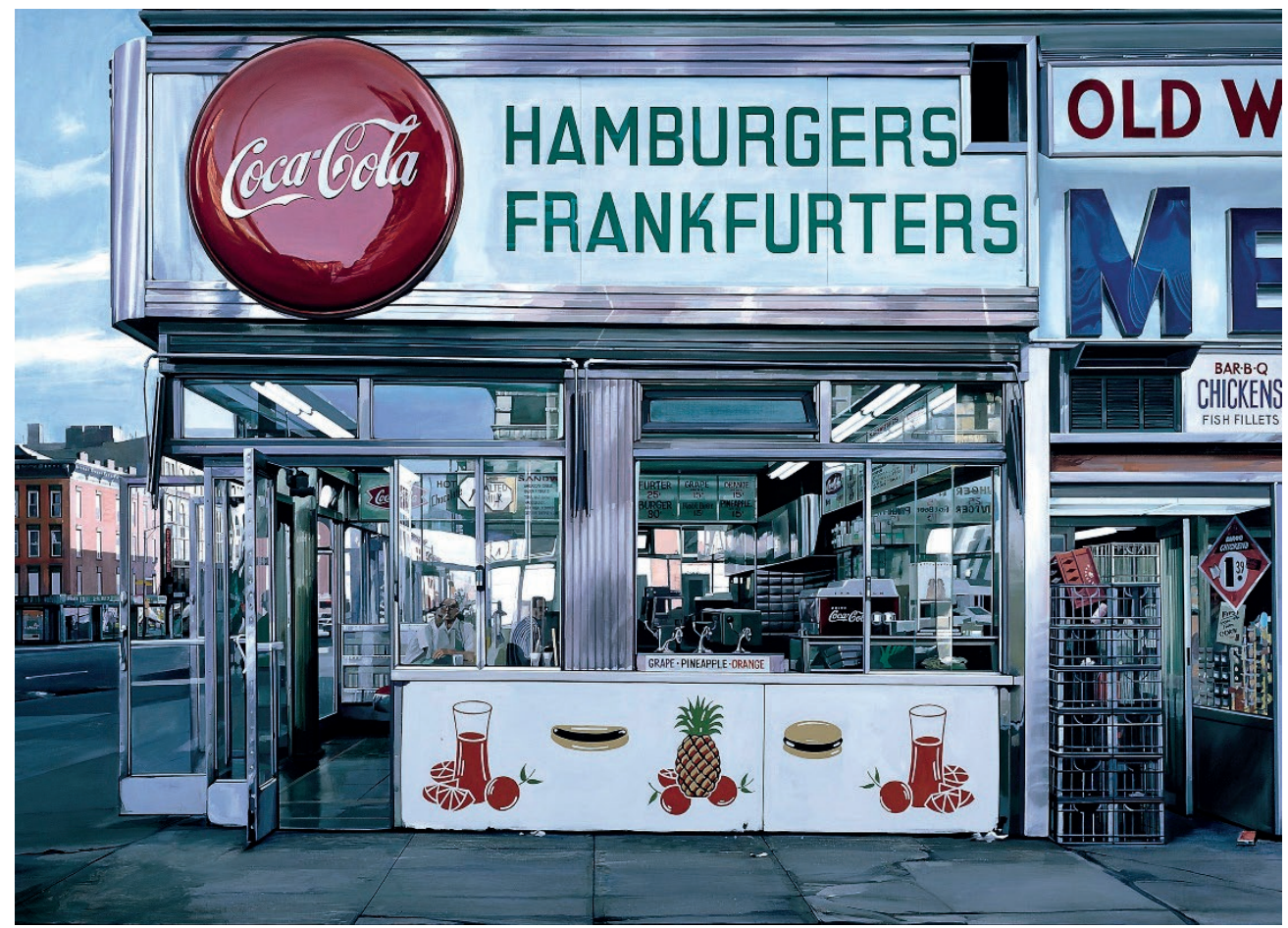

Figura 2. Imagen de Nedick's, de Richard Estes (1970).

Fuente: Museo Thyssen-Bornemisza. https://www.museothyssen.org/sites/default/files/styles/full_resolution/public/imagen/2017-03/ CTB.1993.10_0.jpg

\subsection{Hamburguesa de piso, de Claes Oldenburg}

La obra de Claes Oldenburg, Hamburguesa de piso, también conocida como Hamburguesa gigante, del año 1962, se trata de una lona estampada, rellena de gomaespuma, de dimensiones 132 x $213 \mathrm{~cm}$, y que se encuentra en el Art Gallery of Ontario, en Toronto, Canadá.

Representa un superlativo de consumo de comida rápida, la hamburguesa, a través de una imagen exageradamente grande que provoca incredulidad y rechazo. Es un ejemplo de imagen kitsch, y representa la cultura americana de la comida rápida o fast-food. Una de sus cualidades es que se trata de una escultura "blanda" y no rígida (Figura 3).

\subsection{Pasteles, de Wayne Thibaud}

La obra de Wayne Thibaud, denominada Cake (pasteles), se realizó en el año 1963, y se encuentra en la National Gallery, Washington D.C., Estados Unidos. La técnica es óleo y las dimensiones $152 \times 183 \mathrm{~cm}$. (Figura 4).

Se trata de una obra realista que se enmarca dentro de la tendencia colorista, en la que se muestran pasteles con volumen y asemejados a la imagen real de los mismos. Se diferencia de la obra de Warhol y Lichtenstein porque no se presenta de forma mecánica una imagen, sino una imagen de alimentos que incrementa el deseo de consumo. Muestra, a modo de un escaparate 


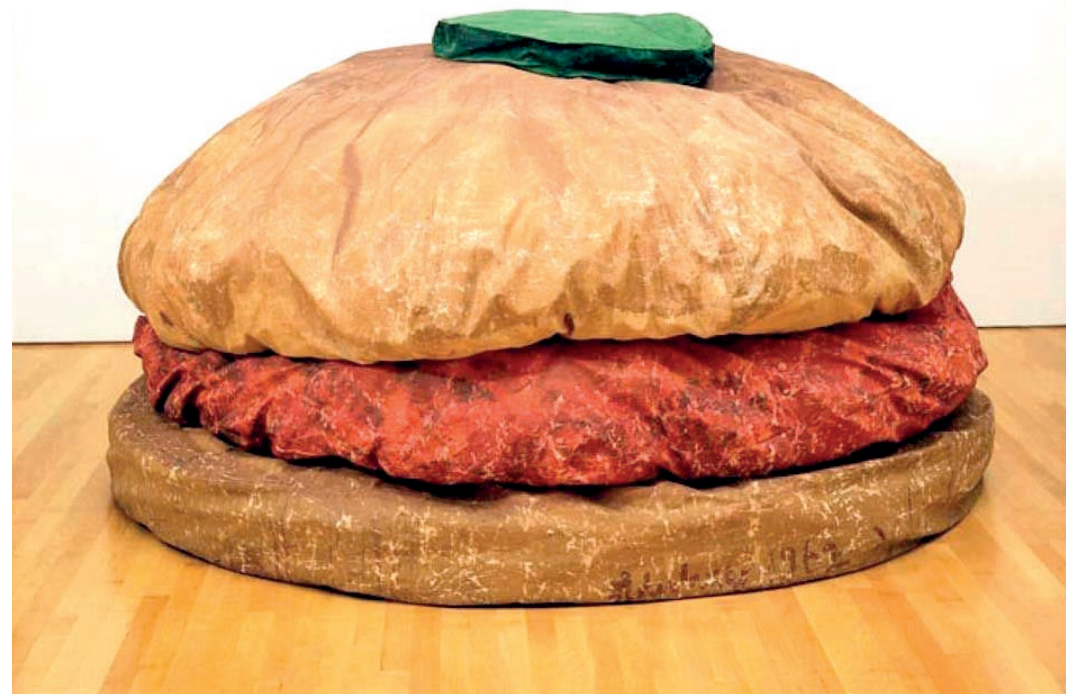

Figura 3. Imagen de Hamburguesa de piso, de Claes Oldenburg (1962).

Fuente: Art \& Life. http://theartandlife.blogspot.com/2012/11un-paseo-por-el-arte-hamburguesa-gigante.html

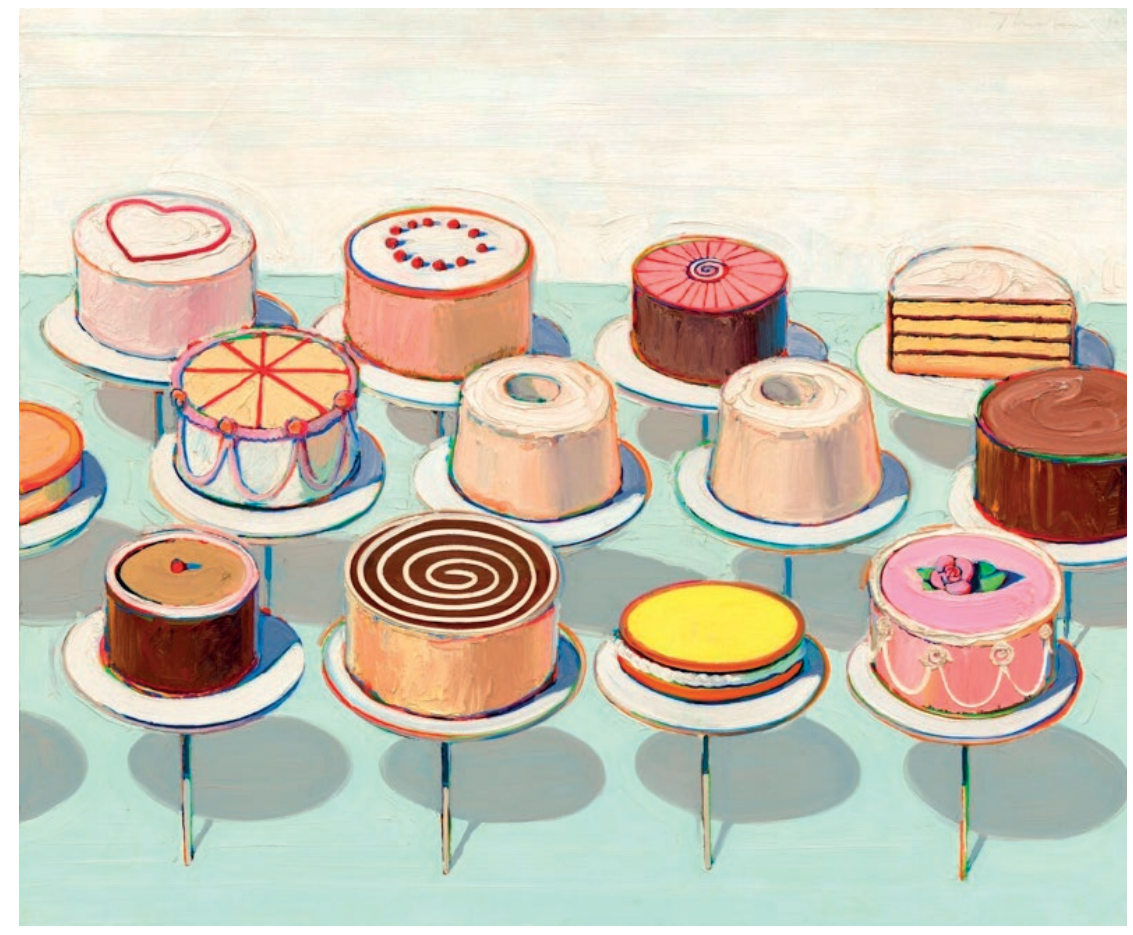

Figura 4. Imagen de Cakes, de Wayne Thibaud (1963).

Fuente: Historia con arte. https://historia-arte.com/obras/pasteles 
de una pastelería, y encima de los soportes, distintos pasteles recubiertos de crema y adornados de guindas y rosas, con excepción de un pastel que ya está cortado, mostrando su interior relleno de chocolate el bizcocho.

Se relaciona con la idea americana de la celebración, y con las formas geométricas incluyendo símbolos como corazones y espirales. Los platos que muestran los dulces parecen suspendidos en el aire, que suma un componente etéreo al conjunto.

\subsection{Sándwich de jamón y huevo, de Tjalf Sparnaay}

Setratadeunaobrahiperrealistadelartistaholandés, TjalfSparnaay (https://www.tjalfsparnaay.nl/). Representa en este caso a un sándwich de jamón y huevo y se inspira en las naturalezas muertas, pero con una técnica muy perfeccionada que muestra los alimentos con una realidad asombrosa (Figura 5). Llega a confundirse su obra pictórica con una imagen fotográfica por la perfección de los detalles. Los colores que utiliza son muy saturados y la obra alcanza unos límites reales altamente conseguidos.

Se enmarca también en la comida del futuro, comida rápida siguiendo el ejemplo de las anteriores obras seleccionadas.

La obra Figura con carne, de Francis Bacon data del año 1954 y refleja un estilo caracterizado por la deformación del personaje, en este caso, el Papa Inocencio X, que lo representa tras de

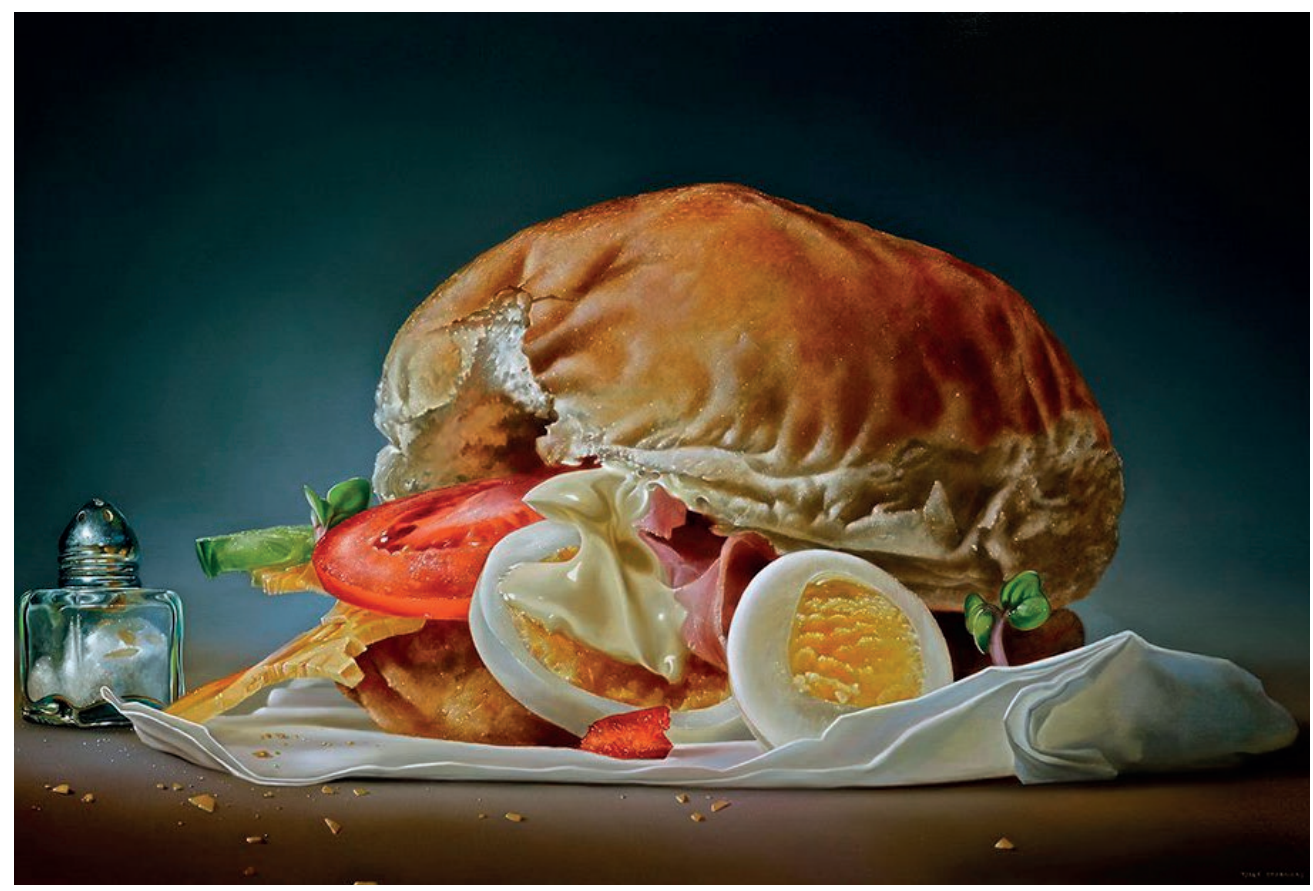

Figura 5. Sándwich de jamón y huevo, de Tjalf Sparnaay (2014).

Fuente: El Universal. https://www.eluniversal.com.mx/menu/obras-de-arte-iconicas-sobre-comida 
sí con dos piezas de carne de vaca (Figura 6). Se encuentra la obra en el Instituto de Arte de Chicago, Chicago, Illinois, Estados Unidos.

Esta obra pictórica muestra el alimento en un segundo plano, mostrando la descarnación de la pieza, y especialmente con las costillas sobresaliendo con una luminosidad que contrasta con la oscuridad del personaje. La presencia de la carne con la figura Papal es de un gran contraste y muestra la relación entre lo sagrado y lo carnal.

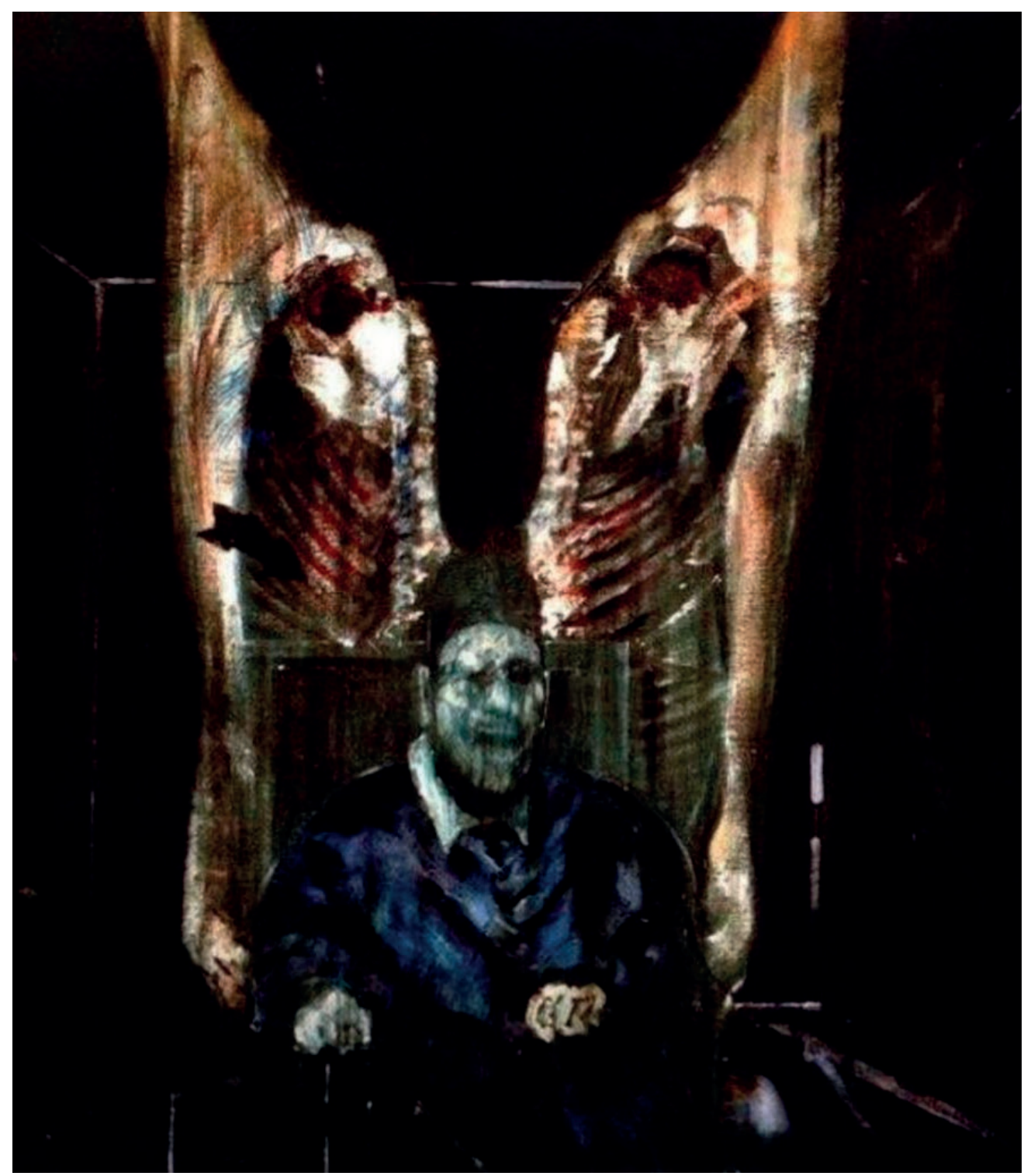

Figura 6. Figura con carne, de Francis Bacon (1954).

Fuente: https://es.wahooart.com/@@/6E3SVB-Francis-Bacon-figura-con-carne-1954- 


\section{Desarrollo de la Innovación: su aplicación a la asignatura Derecho alimentario y protección del consumidor en el Grado en Ciencia y Tecnología de los alimentos, en la Universitat Politècnica de València}

El estudio de los alimentos y su presencia en el arte puede tener una gran aplicación al ámbito de la docencia para mostrar al alumnado la evolución de la presencia de los alimentos en el arte. En el caso de la asignatura de Derecho alimentario y protección del consumidor, que se imparte en el Grado en Ciencia y Tecnología de los alimentos, con una carga docente de $4.5 \mathrm{cr}$. ECTS se puede realizar una actividad enfocada a que el alumnado selecciones obras de arte en la que aparezcan alimentos, que puedan estar relacionados con la ciencia ficción, y ver el tratamiento que el/la artista concede a la representación gráfica. Mediante esta actividad se lograrían diversos objetivos:

a. Conseguir una involucración del alumnado en la búsqueda y conocimiento del arte aplicado a la asignatura;

b. Determinar qué tipo de alimentos aparecen en una obra artística y su razón de ser, para analizar la conexión en relación con el tiempo de la obra y los aspectos sociales que tienen los alimentos (por ejemplo, vino, dulces, etc.).

c. Mostrar la evolución en el consumo de los alimentos a través de las distintas épocas históricas (por ejemplo, durante la mitad del siglo XX se incrementó el consumo de bebidas refrescantes asociadas a la felicidad o bienestar).

Esta actividad consistiría en formar distintos grupos de alumnado que seleccionarán distintas obras de arte en las que esté presente el alimento, y se determinarán sus características y peculiaridades. Cada alumno/a rellenará una ficha facilitada por la profesora en la que explicará sus experiencias, así como las motivaciones que le sugiere la obra en relación con el alimento (funcionalidad, relación social, características del mismo, y componentes nutricionales).

Al finalizar la actividad, se realizará un foro de debate en que cada grupo explicará la obra seleccionada y los alimentos que aparecen en la misma, motivando las opiniones formuladas.

\section{Conclusiones}

Los alimentos y el arte han estado estrechamente vinculados a lo largo de los siglos. Son numerosos los ejemplos que podemos extraer de la representación de los alimentos en las obras, principalmente pictóricas. Abundan en los museos las pinturas de bodegones, de naturaleza muerta, que han sido representados en las diversas corrientes artísticas.

En el caso de los alimentos del futuro, entendiendo por tales alimentos que han supuesto un consumo en las nuevas generaciones, y que han estado influidos por la ciencia ficción a través de diversos hitos, por ejemplo, cinematográficos, nos encontramos con la representación de la denominada comida rápida o fast food. El hiperrealismo también ha contribuido a plasmar en la obra de los artistas pertenecientes a este movimiento la comida americana, representada por refrescos, sándwiches, y también los pasteles altamente edulcorados y con una visión atractiva para el consumidor. 
Se echa en falta la representación de los denominados nuevos alimentos, alimentos obtenidos por las tecnologías, que no han sido objeto de tratamiento pictórico, pero que en un futuro podrán ser objeto de atención por parte de los artistas.

Todos los ejemplos que hemos elegido para representar el futurismo, se encuentran expuestos en los museos, y forman parte de la creación artística de pintores que han plasmado el alimento en relación a su visión del mundo, por ejemplo, en el caso de Francis Bacon, cuya enigmática obra relaciona la figura de un Papa, con dos pedazos de carne de vacuno.

La exposición de las obras y su análisis puede ser también utilizado en el ámbito docente, a través de una actividad en la que el alumnado reflexiona sobre la representación de los alimentos a lo largo del tiempo.

\section{Agradecimientos}

Trabajo realizado en el marco del Proyecto de Innovación y Mejora Educativa (PIMEs) "Gamificación y TICs: diseño de actividades audiovisuales basadas en la ciencia ficción para la dinamización docente en un entorno presencial, semipresencial y virtual", presentado en el marco de la convocatoria de Proyectos de Innovación y Mejora Educativa (PIMEs) llevada a cabo en la Universitat Politècnica de València para el curso 2020-2022 obteniendo resolución favorable de la Comisión de Evaluación y Seguimiento de Proyectos de Innovación y Convergencia (CESPIC) en su sesión de septiembre de 2020 y concedido por el Vicerrectorado de Estudios y Convergencia Europea de la Universitat Politècnica de València. Años 2020-2022. Investigadora principal: Francisca Ramón Fernández. y en el marco del Proyecto I+D+i «Retos investigación» del Programa estatal de I+D+i orientado a los Retos de la Sociedad del Ministerio de Ciencia, Innovación y Universidades: RTI2018-097354-B-100 (2019-2022).

\section{Bibliografía}

DÍAZ RUIZ, Ma. DEL C. (2017). Arte y comida en la creación contemporánea desde un enfoque de género. Málaga: Universidad de Málaga.

FUCHS, L. (2021). "Ángel León anuncia su último gran descubrimiento: un cereal marino sostenible cultivado por primera vez en Aponiente". Directo al paladar. El sabor de la vida. https://www. directoalpaladar.com/actualidad-1/nuevo-alimento-revolucionario-angel-leon-cereal-marinosostenible-cultivado-primera-vez-aponiente [Consulta: 16 de septiembre de 2021].

MIÑO, M. A. (2020). "Desde las vanguardias al arte contemporáneo latinoamericano: la comida como elemento transformador". En: Index. Revista de arte contemporáneo, núm. 1, pp. 60-66. http://www.revistaindex.net/index.php/cav/article/view/330 [Consulta: 18 de septiembre de 2021].

NATIONAL GEOGRAPHIC (2016). Verduras en Marte: cada vez más cerca de la comida marciana. https://www.nationalgeographic.es/espacio/verduras-en-marte-cada-vez-mas-cerca-de-lacomida-marciana [Consulta: 16 de septiembre de 2021]. 